\title{
Strain-Induced Spectral Tuning of the Whispering Gallery Modes in a Cylindrical Micro-Resonator Formed by a Polymer Optical Fiber
}

\author{
Arun Mallik \\ Technological University Dublin, arun.mallik@tudublin.ie \\ Vishnu Kavungal \\ Technological University Dublin, vishnu.kavungal@tudublin.ie \\ Yuliya Semenova \\ Technological University Dublin, yuliya.semenova@tudublin.ie
}

See next page for additional authors

Follow this and additional works at: https://arrow.tudublin.ie/engschmanconn

Part of the Engineering Commons

\section{Recommended Citation}

Kavungal, V., Mallik, A. \& Semenova, Y. (2017). Strain-induced spectral tuning of the whispering gallery modes in a cylindrical micro-resonator formed by a polymer optical fiber. Applied Optics, vol. 56, no. 5. doi.org/10.1364/A0.56.001339

This Article is brought to you for free and open access by the School of Manufacturing and Design Engineering at ARROW@TU Dublin. It has been accepted for inclusion in Conference Papers by an authorized administrator of ARROW@TU Dublin. For more information, please contact arrow.admin@tudublin.ie, aisling.coyne@tudublin.ie,gerard.connolly@tudublin.ie.

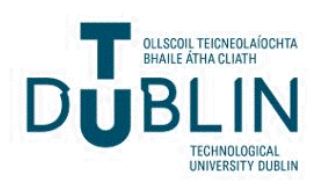




\section{Authors}

Arun Mallik, Vishnu Kavungal, Yuliya Semenova, and Qiang Wu

This article is available at ARROW@TU Dublin: https://arrow.tudublin.ie/engschmanconn/44 


\title{
applied optics
}

\section{Strain-induced spectral tuning of the whispering gallery modes in a cylindrical micro-resonator formed by a polymer optical fiber}

\author{
Vishnu Kavungal, ${ }^{1, *}$ Arun Kumar Mallik, ${ }^{1}$ Gerald Farrell, ${ }^{1}$ Qiang Wu, ${ }^{1,2}$ and Yuliya Semenova ${ }^{1}$ \\ ${ }^{1}$ Photonics Research Centre, Dublin Institute of Technology, Kevin Str., Dublin, Ireland \\ ${ }^{2}$ Department of Mathematics, Physics and Electrical Engineering, Northumbria University, Newcastle upon Tyne NE1 8ST, UK \\ ${ }^{*}$ Corresponding author: vishnu.kavungal@mydit.ie
}

Received 30 November 2016; revised 10 January 2017; accepted 12 January 2017; posted 13 January 2017 (Doc. ID 281878); published 3 February 2017

\begin{abstract}
A mechanical strain assisted technique for spectral tuning of whispering gallery modes (WGM) in a cylindrical micro-resonator formed by a polymer optical fiber (POF) is investigated. WGMs in the POF-based micro-cylinder are excited by evanescent light coupling using a tapered single mode silica fiber. WGMs observed in the transmission spectrum of the silica fiber taper have a high extinction ratio of up to $19 \mathrm{~dB}$ and a quality factor ( $Q$-factor) of up to $2.64 \times 10^{4}$. The application of tensile axial strain $(\mu \varepsilon)$ in the range from 0 to $1746 \mu \varepsilon(0.17 \%)$ to the POF micro-resonator results in a linear shift of the WGM spectrum with a sensitivity of $0.66 \mathrm{pm} / \mu \varepsilon$. Under the influence of the applied strain, the WGMs undergo a blue shift and return to their initial spectral positions after the strain is decreased. The proposed strain tunable POF micro-resonator has potential applications in fiber optic sensing and tunable micro lasers. (c) 2017 Optical Society of America
\end{abstract}

OCIS codes: (140.3945) Microcavities; (140.3948) Microcavity devices; (060.2370) Fiber optics sensors; (250.5460) Polymer waveguides.

\section{INTRODUCTION}

Whispering gallery mode (WGM) micro-resonators (MRs) are attractive photonic devices due to their small mode field volume, narrow spectral linewidths, and high $Q$-factors [1-3]. Such MRs have found applications in numerous fields, including studies of nonlinear optical effects [4], in quantum electrodynamics [5], and for low threshold miniature scale lasers [6]. One of the most promising applications of WGM MRs is in the area of optical sensing $[2,7,8]$, where such resonators exhibit a higher sensitivity than their macro-fiber counterparts. WGMs can be supported by a variety of resonator geometries, such as spheres [9], spheroids [10], disks [11], rings [8], and cylinders [12]. The choice of the specific resonator for a given application is typically impacted by three main considerations, such as the maximum achievable $Q$-factor, simplicity of fabrication, and ease of interconnection [13].

For cylindrical resonators based on a section of optical fiber, there are a number of potential advantages that make such resonators worth exploring: (1) a very simple fabrication process, since optical fibers are highly uniform in diameter, allowing large numbers of identical resonators to be fabricated and providing a high degree of repeatability; (2) optical fibers can be easily manipulated and mounted; and (3) the alignment for optimal coupling of the excitation light into the fiber MR has on only one angular degree of freedom, as opposed to two for experiments involving microspheres [14]. As a result, the optical setup for the cylindrical fiber-based MR experiments is more straightforward.

Spectral positions of WGM resonances can be tuned by various external stimuli. Numerous reports demonstrate the tuning of WGM resonant wavelengths of spherical MRs by changing the surrounding refractive index [15], applying mechanical strain to the resonator [16], changing internal pressure [17], applying external electric field [18], etc. Self-tuning of WGM resonances has been demonstrated for a stand-alone silica microsphere by changing its temperature under the influence of the gas flow surrounding the resonator [19]. In a cylindrical MR, tuning of WGM resonances using mechanical strain was demonstrated for the first time by Huston and Eversole [20]. In this work, the morphology dependent resonance properties were studied in the elastic scattering spectra of a $1 \mathrm{~mm}$ long section of a $125 \mu \mathrm{m}$ diameter single mode silica optical fiber.

Due to their relatively high elastic modules, silica WGM resonator-based sensors have limited sensitivity to strain or force. Measurement sensitivity can be improved by using 
dielectric materials with smaller elastic modulus, such as polymers. For example, Ioppolo et al. [21-24] have demonstrated that solid, as well as hollow polymethyl-methacrylate (PMMA), microspheres have a higher strain sensitivity compared to those made of silica.

More recently, the tuning of WGM lasing modes in a polymer optical fiber (POF) -based resonator under tensile strain was reported in [25]. The experiment was carried out by the scattering of focused laser beams on a section of Rhodamine-B-doped POF. The spectral shift of WGMs excited in a photonic crystal fiber MR infiltrated with magnetic fluid under the influence of external magnetic fields is investigated in [26], and the laser assisted tuning method for WGMs in a cylindrical MR based on a magnetic-fluids infiltrated microstructured optical fiber is reported in [27].

This paper presents the findings of a comprehensive investigation of mechanical strain tuning of WGMs for a POF-based cylindricalMR. Cross coupling takes place between the evanescent fields of the guided modes from a tapered single mode silica optical fiber in close physical contact with a microcylinder with an outer diameter of $490 \mu \mathrm{m}$. The transmission spectrum of the silica taper coupled to the POF MR shows very high extinction ratio dips, corresponding to the WGM eigenmodes. Spectral tuning of the WGM dips is investigated under the influence of increasing and decreasing axial tensile strain that is applied to the POF micro-cylinder. The influence of the input light polarization on the WGM's tuning is also studied by carrying out the experiments for different input light polarization states.

The proposed POF cylindrical MR possesses several desirable features, such as highly sensitive strain assisted tunability, high linear strain dependence, and ease of fabrication, which make it a good candidate for applications in optical fiber sensing and tunable micro-fiber lasing.

\section{EXPERIMENTAL ARRANGEMENTS}

The experimental setup for studies of the influence of strain on the WGMs excited in the cylindrical MR is schematically shown in Fig. 1. The cylindrical MR was formed by a section of a $490 \pm 5 \mu \mathrm{m}$ (outer diameter) graded index POF (GIPOF 50, Thorlabs). The evanescent light coupling to the microcylinder was achieved by a full fiber taper placed in close

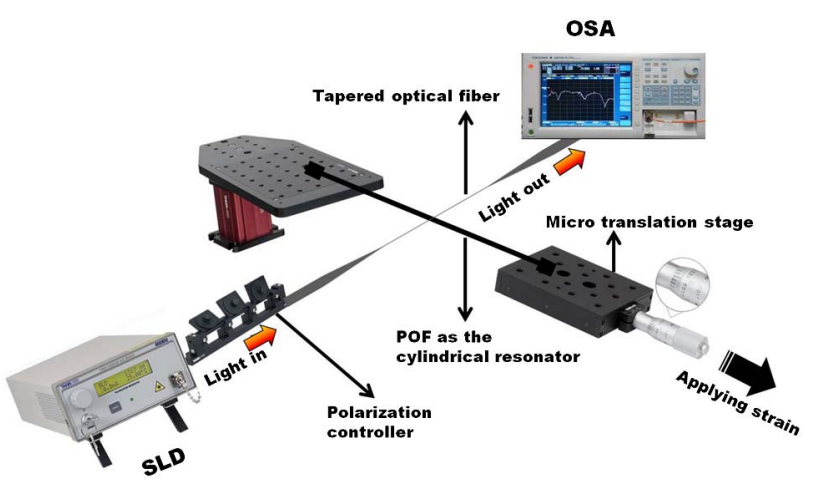

Fig. 1. Schematic diagram of the experimental setup for studies of the tunability of WGMs in the POF resonator under the influence of applied strain. contact with the POF micro-cylinder [28]. The optical fiber tapering is undertaken using the micro heater brushing technique [29]. The fabricated fiber taper has a waist diameter of circa $1.3 \mu \mathrm{m}$. The input end of the fiber taper was connected to a super luminescent diode (SLD) (Thorlabs) with a wavelength range of 1500-1600 $\mathrm{nm}$. Light from the SLD was passed through a three-paddle manual polarization controller, and the output of the fiber taper was connected to the optical spectrum analyzer (OSA) (86142B, Agilent).

As illustrated in Fig. 1, in order to apply the strain to the resonator, one end of the POF micro-cylinder was immobilized with a fiber holder, and the other end was attached to the micro translation stage with a resolution of $10 \mu \mathrm{m}$. The length between the two fixed points is considered as the sensing length. Here, the sensing length of the POF MR was set to $63 \mathrm{~mm}$ for ease of mounting. The strain is applied to the POF microcylinder by moving the translation stage with a step size of $10 \mu \mathrm{m}$ [corresponding to an applied tensile strain step of $159 \mu \varepsilon(0.016 \%)]$ in a direction away from the fixed end.

During each experiment, the polarization state of the input light was controlled by the three-paddle polarization controller (FPC030, Thorlabs). For investigation of the effect of the input light polarization on the WGM's tuning properties, we compare our results for three different linear polarization states, as described later in this paper. During each measurement of the shift of the WGM spectrum with respect to the applied strain, the input polarization state was fixed. All of the measurements were carried out at a constant laboratory temperature $\left(20^{\circ} \mathrm{C}\right)$.

\section{A. Effect of Strain on the WGM Resonance Wavelength}

Figure 2(a) shows the WGM spectrum of the unstrained POF micro-cylinder with a diameter of $490 \mu \mathrm{m}$. Three WGM resonance dips are observed within the selected 3.6-nm-wide wavelength band. The full widths at half-maxima (FWHMs) of the observed resonance dips are measured, and their $Q$-factors are calculated using the equation $Q=\lambda / \Delta \lambda$, where $\lambda$ is the resonance wavelength, and $\Delta \lambda$ is the corresponding FWHM. All of the $Q$-factors of the resonance dips in the selected range are approximately equal to $2 \times 10^{4}$. For example, Fig. 2(b) shows the Lorentz fit of a selected resonance dip (p1). The dip has the central wavelength at $1500.71 \mathrm{~nm}$, and its FWHM and the corresponding $Q$-factor are $0.072 \mathrm{~nm}$ and $2.09 \times 10^{4}$, respectively. The extinction ratios of all three of the resonant dips are larger than $18 \mathrm{~dB}$. The average free spectral range associated with the resonant pattern in the specified wavelength range is $1.07 \mathrm{~nm}$.

We experimentally investigated the strain tuning of the POF-based MR using the experimental setup schematically illustrated in Fig. 1. Figure 3 shows the evolution of the WGM spectrum of the POF MR as the axial strain is increased from zero to $1746 \mu \varepsilon$ and then decreased back to zero. As the axial strain increases, the resonant dips exhibit a linear blue shift. When the applied tensile strain is decreased back to zero, the resonance dips linearly red shift back to their initial positions, which indicates a good reversibility of the proposed WGM tuning approach.

Before considering the wavelength tuning in more detail, it is useful to analyze the expected tuning effect of strain. For this, 

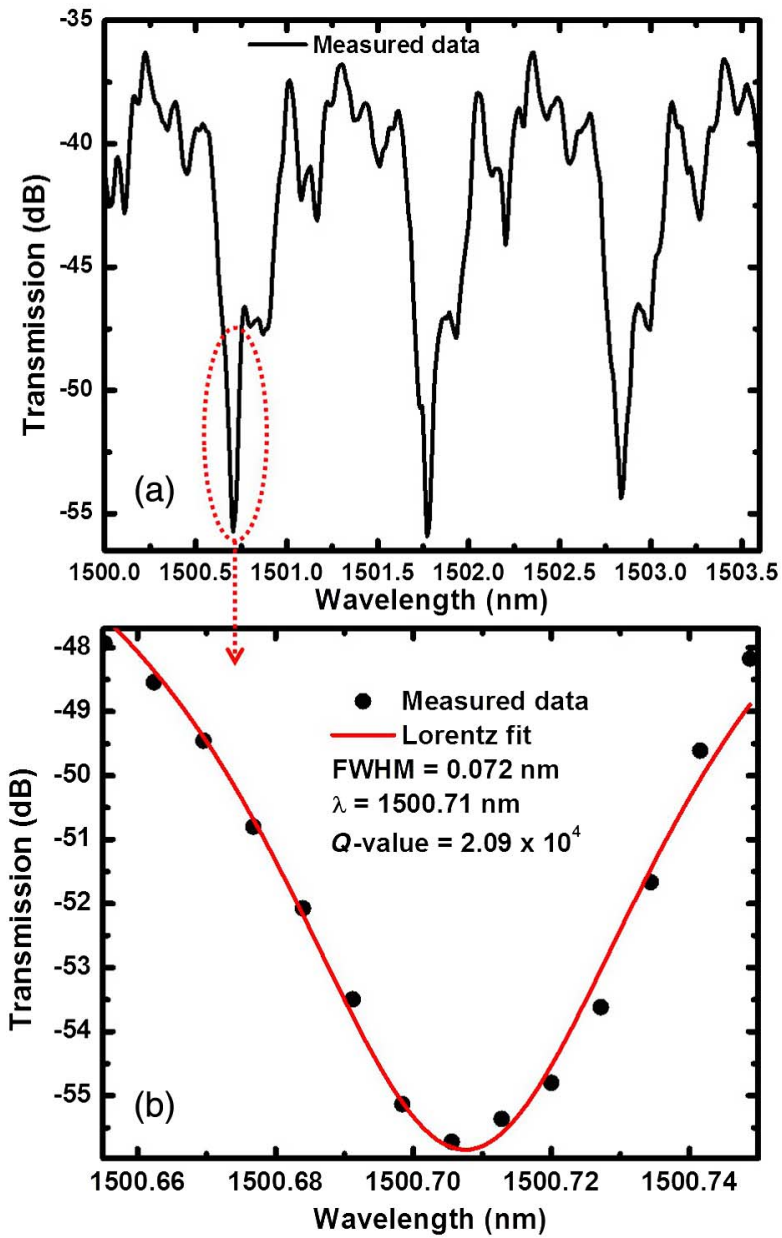

Fig. 2. (a) WGM's spectrum generated by the POF MR, (b) Lorentzian fittings of the spectral dips p1. The dip has the central wavelength at $1500.71 \mathrm{~nm}$, and its FWHM and the corresponding $Q$-factor are $0.072 \mathrm{~nm}$ and $2.09 \times 10^{4}$, respectively.

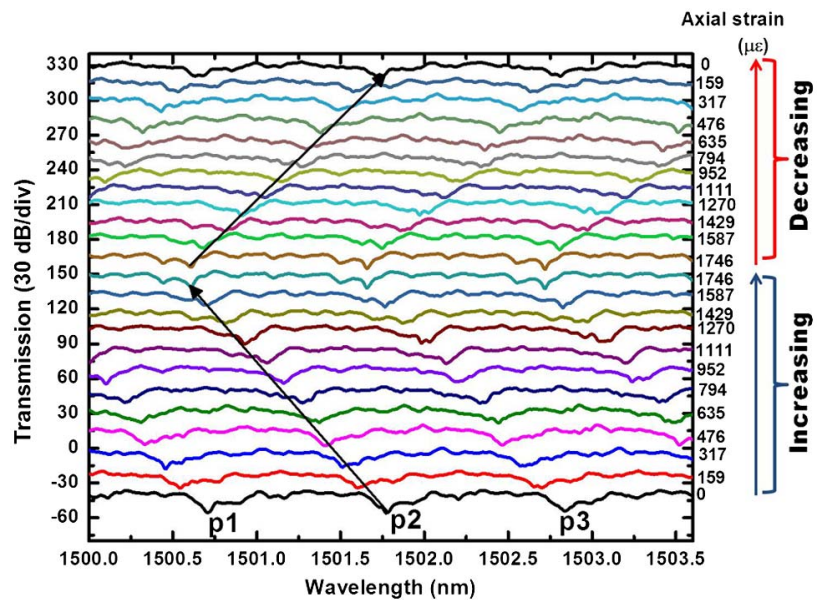

Fig. 3. Evolution of the tapered fiber transmission spectrum under the influence of the increasing axial strain, followed by the strain decrease back to the unstrained position. For illustrative purposes, each consecutive spectrum is plotted with an artificial offset of $30 \mathrm{~dB}$ compared to the prior spectrum. The arrows indicate the resonant wavelength shift direction. a simple model to predict the tuning of the WGM resonances was developed based on the two primary assumptions as follows: the first one is the fact that the MR diameter can be related to the value of the applied axial tensile strain. The second assumption is that there is a measurable change in the refractive index brought about by the different mechanical stress and strain components within the resonator material. Taken together, the fractional change in the wavelength of the WGMs is [21-25,30-32]

$$
\frac{\Delta \lambda}{\lambda}=\frac{\Delta D}{D}+\frac{\Delta n}{n},
$$

where $\Delta D / D$ is the fractional change in the MR diameter $(D)$ due to the deformation of the micro-cavity, and $\Delta n / n$ is the fractional change in the refractive index $(n)$ due to the induced stress. The transverse deformation $(\Delta D / D)$ of the micro-cavity can be related to the axially applied tensile strain $(\Delta L / L)$ by the Poisson ratio $(\sigma)$. The Poisson ratio is the ratio of the lateral contraction strain $(\Delta D / D)$ to the axial tensile strain $(\Delta L / L)$ :

$$
\sigma=\frac{\Delta D / D}{\Delta L / L}, \frac{\Delta D}{D}=\sigma \frac{\Delta L}{L} .
$$

Changes in the fiber dimensions result in changes in the material density with the corresponding induced changes in its refractive index $(\Delta n)$. Thus, changes in the refractive index can be expressed as

$$
\Delta n=n P_{\text {eff }} \frac{\Delta L}{L},
$$

where $P_{\text {eff }}$ is the effective strain-optic coefficient $[25,32]$. Subsequently, the WGM resonance wavelength shift can be expressed as

$$
\frac{\Delta \lambda}{\lambda}=-\left(\sigma \frac{\Delta L}{L}+P_{\mathrm{eff}} \frac{\Delta L}{L}\right) .
$$

Here, the negative sign shows that the axial tensile strain applied to the POF MR will result in a blue shift of the WGM resonance. The Poisson ratio and the effective strain-optic coefficient of PMMA are $0.35-0.45$ and 0.099 $( \pm 0.0009)$, respectively, $[20,25,32,33]$. By assuming the Poisson's ratio $(\sigma)$ of the POF is 0.35 , the theoretical calculation based on Eq. (4) shows that the sensitivity of the WGM resonance wavelength shift with respect to the increasing and decreasing tensile strain is $0.68 \mathrm{pm} / \mu \varepsilon$.

Figures 4(a) and 4(b) correspond to the resonance dips p2 and $\mathrm{p} 3$, respectively (as depicted in Fig. 1). Here, the black dots are the measured data, and the solid red line is the linear fit. It is clear that all of the resonance dips move toward the shorter wavelengths in a linear fashion with a good sensitivity to the applied axial tensile strain. For each $10 \mu \mathrm{m}$ axial elongation [corresponding to a tensile strain of $159 \mu \varepsilon(0.016 \%)$ ], it is found that the WGMs shift by $0.11 \mathrm{~nm}$ on average. Here, the second dip of the WGM spectrum, p2, shifts from 1501.77 to $1500.605 \mathrm{~nm}$, and the third dip, p3, shifts from 1502.84 to $1501.66 \mathrm{~nm}$ in response to a $110 \mu \mathrm{m}$ elongation [corresponding to the applied strain of $1746 \mu \varepsilon(0.17 \%)$ ]. Linear fitting of the wavelength response data indicates that the linear regression coefficient is greater than 0.99 for both $\mathrm{p} 2$ and $\mathrm{p} 3$ dips. It could also be seen that the tuning sensitivities 

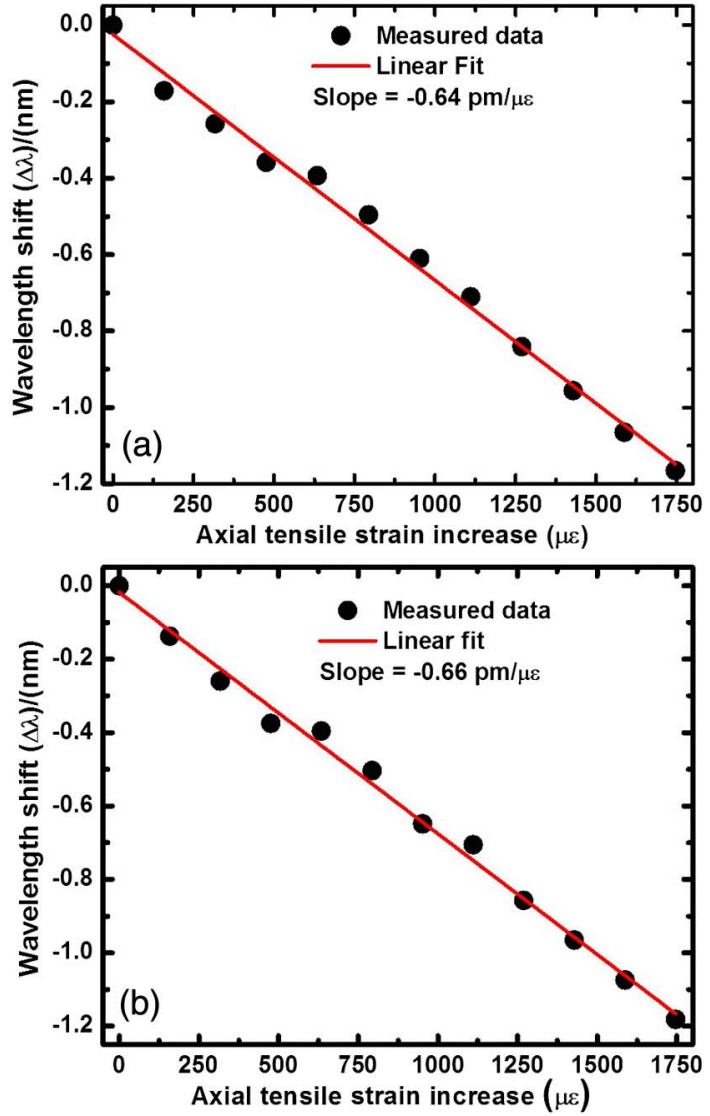

Fig. 4. Wavelength shift of the selected WGM resonances as a function of increasing axial strain: (a) dip p2 and (b) dip p3 (as depicted in Fig. 2). The measured wavelength shift (scatter data) is linearly fitted (solid line). Linear fitting of the wavelength response data indicates that the linear regression coefficient is greater than 0.99 for both p2 and p3 dips. The slopes of the linear dependencies are $-0.64 \mathrm{pm} / \mu \varepsilon$ for $\mathrm{p} 2$ and $-0.66 \mathrm{pm} / \mu \varepsilon$ for $\mathrm{p} 3$, respectively.

of these WGMs are equal to 0.64 and $0.66 \mathrm{pm} / \mu \varepsilon$ for $\mathrm{p} 2$ and p3 dips, respectively, which is in close agreement with the theoretically calculated sensitivity of $0.68 \mathrm{pm} / \mu \varepsilon$.

The wavelength tuning of the WGM of the POF MR by applying axial tensile strain is also found to be reversible. Figure 5 shows the return of the shifted spectra to their initial positions with the decrease of the applied axial tensile strain.

Figures 5(a) and 5(b) illustrate the wavelength shift corresponding to the decrease of the applied axial tensile strain for the WGM resonant dips $\mathrm{p} 2$ and $\mathrm{p} 3$, respectively. Here, the black dots are the experimentally measured data, and the solid red line is the linear fit. Here, the linear regression coefficient is greater than 0.99 for both $\mathrm{p} 2$ and $\mathrm{p} 3$ dips. The negative values of the $\mathrm{X}$ axis illustrate the gradual decrease of the applied axial tensile strain from its maximum value to the initial unstrained state. It is clear that all of the resonance dips move toward the longer wavelengths (initial position) in a linear fashion. For each $10 \mu \mathrm{m}$ translation [corresponding to the decrease in the applied strain by $159 \mu \varepsilon(0.016 \%)]$ in the axial direction, it is found that the WGMs experience an average red shift of $0.11 \mathrm{~nm}$. That means that the spectral shifts of the WGMs are
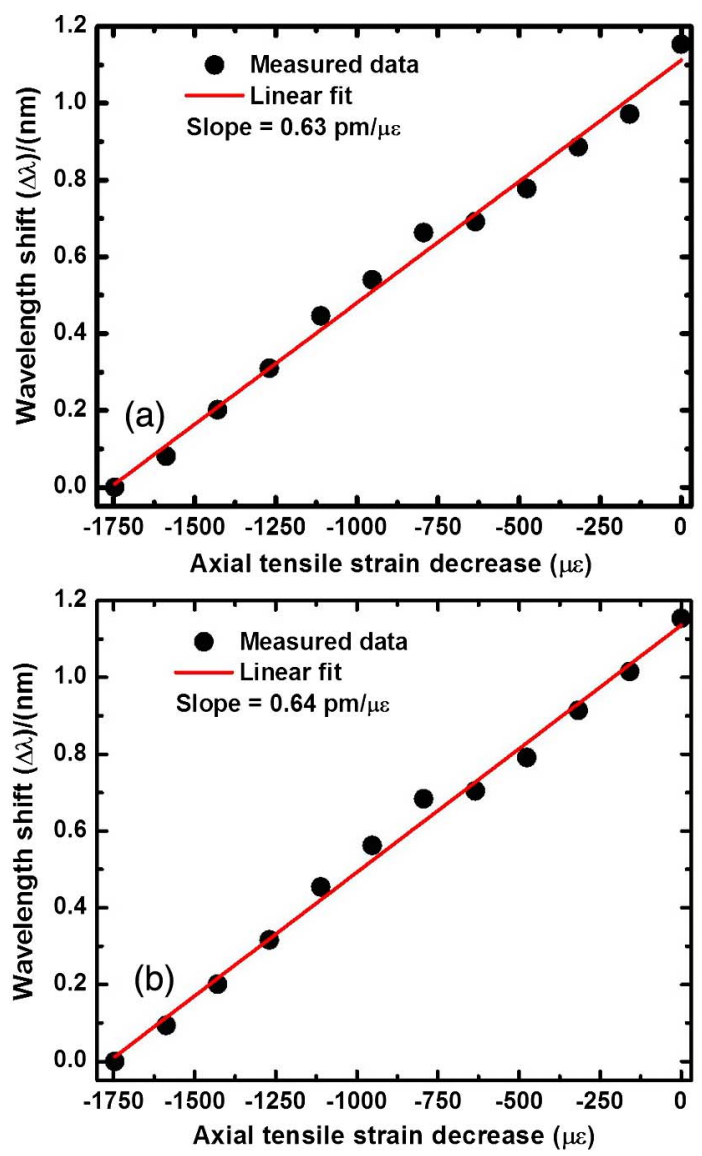

Fig. 5. Wavelength shifts of the selected WGM resonances as a function of decreasing axial strain: (a) dip p2 and (b) dip p3. The measured wavelength shift (scatter data) is linearly fitted (solid line). Linear fitting of the wavelength response data indicates that the linear regression coefficient is greater than 0.99 for both $\mathrm{p} 2$ and $\mathrm{p} 3$ dips. The slopes of the linear dependences are $0.63 \mathrm{pm} / \mu \varepsilon$ for $\mathrm{p} 2$ and $0.64 \mathrm{pm} / \mu \varepsilon$ for p3, respectively.

similar in both cases with the increase and decrease of the applied axial tensile strain. Here, the second dip of the WGM spectrum, p2, shifts from 1500.60 to $1501.76 \mathrm{~nm}$, and the third dip, p3, shifts from 1501.66 to $1502.81 \mathrm{~nm}$ over the range of strains from the maximum value of $1746 \mu \varepsilon$ $(0.17 \%)$ to zero. The strain sensitivity of dip p2 is $0.63 \mathrm{pm} / \mu \varepsilon$ and that of $\mathrm{p} 3$ is $0.64 \mathrm{pm} / \mu \varepsilon$, both are in close agreement with the theoretical slope of $0.68 \mathrm{pm} / \mu \varepsilon$.

Detailed studies of the hysteresis of the WGM's tuning with the applied strain and the outcomes are presented in Fig. 6. Figures 6(a) and 6(b) show the WGM's resonance wavelengths of $\mathrm{p} 2$ and $\mathrm{p} 3$ during the full measurement cycle, including the increase in the applied axial tensile strain and its subsequent complete decrease. Here, the black arrows indicate the direction of the WGM's shift during the increase of the applied axial tensile strain, and the red arrows show the direction of the spectral shifts during the strain decrease. The inset graphs in Figs. 6(a) and 6(b) illustrate the differences in the WGM dip wavelengths corresponding to the same strain values applied axially during the strain increase $[\lambda($ S.I $)]$ and strain decrease $[\lambda($ S.D) $]$ cycles. The maximum dip wavelength difference between the increase 


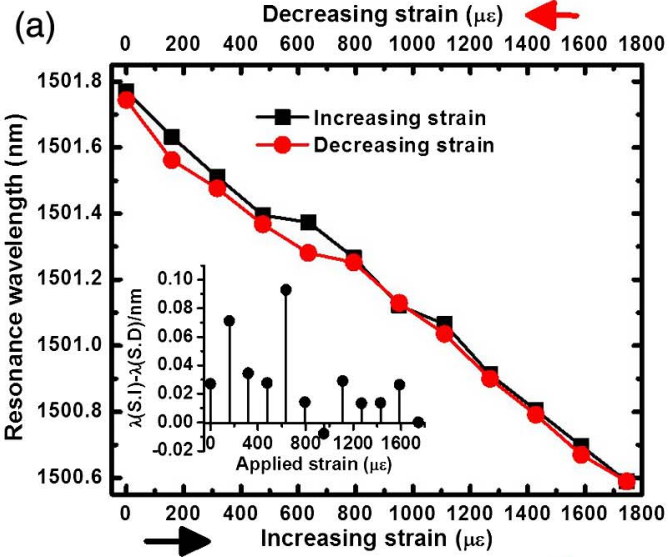

Decreasing strain ( $\mu \varepsilon)$

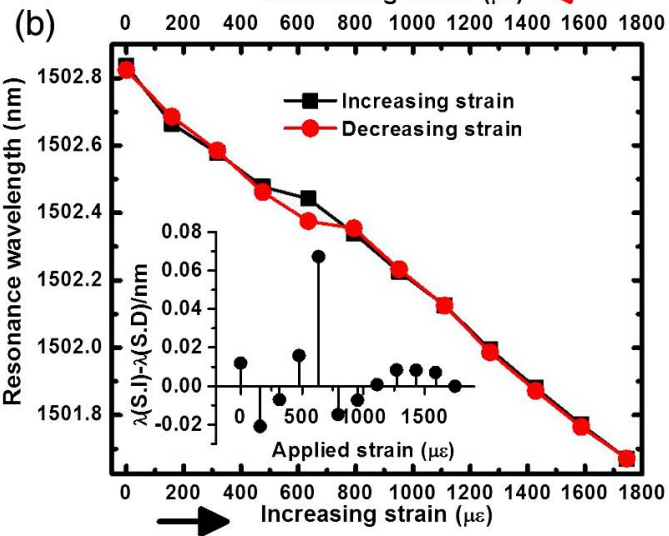

Fig. 6. Selected WGM resonances during the strain increase (blue) and strain decrease (red) cycles: (a) dip p2 and (b) dip p3. Inset figures show the discrepancies in WGM wavelengths during the increase and decrease cycles.

and decrease cycles of the applied axial strain is estimated as $90 \mathrm{pm}$.

Such small discrepancies between the WGM wavelengths when increasing and decreasing the strain could be explained by the nonuniform deformations experienced by the POF micro-cylinder during elastic deformation [25].

\section{B. Polarization Effects}

In order to investigate the impact of the input light polarization and to demonstrate the repeatability of the results presented in the previous section, we carried out the experiments above for different input light polarizations.

As shown previously in Fig. 1, the polarization of the light at the input end of the fiber taper is controlled by the three-paddle polarization controller (FPC030, Thorlabs). The working principle of FPC030 is based on the stress-induced birefringence within single mode fiber (SMF 28) created through the bending and twisting of the fiber.

The quarter wave plate in the polarization controller transforms the input polarization state into a linear polarization state. The linearly polarized light can then be rotated to a desired angle with respect to the initial state using the middle halfwave plate paddle of the polarization controller. The twisting regions of the fiber due to rotating the paddle by an angle " $\tau$ " will rotate the polarization by an angle

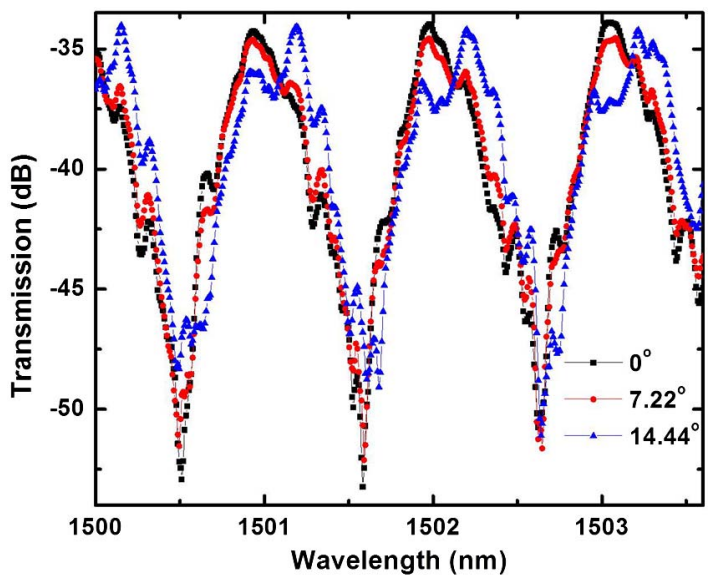

Fig. 7. WGM resonant spectra from the POF MR with different input light polarizations.

$$
\theta=\alpha \tau,
$$

where $\alpha=-n^{2} p_{44}$ [34]. Here, $p_{44}$ is the elasto-optic coefficient of the fiber, and $n$ is the refractive index of the core. The elastooptic coefficient of fused silica is given by $p_{44}=\frac{p_{11}-p_{12}}{2}$, where the numerical values of $p_{11}$ and $p_{12}$, respectively, are given by 0.121 and 0.270 [35].

For the experiments, the input polarization state was set as follows: the three paddles had initial vertical positions, and then the middle half-wave fiber paddle was rotated through its full travel range, while the other two quarter wave fiber paddles remained in their starting vertical positions. The strain tunability experiments using POF MR were repeated for three more linearly polarized input states by rotating by a $0^{\circ}, 45^{\circ}$, and $90^{\circ}$ angle of the central half-wave plate of the polarization controller from its initial vertical position. The corresponding rotation of polarization from the initial position is calculated by Eq. (5) as $0^{\circ}, 7.22^{\circ}$, and $14.44^{\circ}$, respectively.

For comparison, Fig. 7 shows the WGM resonance spectra excited by a $490 \mu \mathrm{m}$ diameter POF MR with the $0^{\circ}, 7.22^{\circ}$, and $14.44^{\circ}$ angles of the input light polarization. The $Q$-factors of the resonance dips are of the same order of magnitude in each of the cases $\left(\sim 10^{4}\right)$. The maximum extinction ratio of the resonances reaches up to $19 \mathrm{~dB}$. The average free spectral range of the resonances is $1.07 \mathrm{~nm}$. With the changes in the input light polarization, the WGM spectra show small differences in the dip amplitudes and shapes, but no significant influence was observed on the tunability of the WGM's spectrum.

Strain sensitivity during the increasing and decreasing cycles of the axial tensile strain shows small differences arising from polarization state changes, possibly because of the nonuniform deformation experienced by the strained POF MR. Figures $8(\mathrm{a})-8(\mathrm{c})$ show the selected WGM resonance wavelengths during the increasing and decreasing cycles of the axial tensile strain for different input polarization orientations.

Figure $8(\mathrm{~d})$ shows the corresponding strain sensitivity of the POF MR undergoing increasing and decreasing strain cycles. 

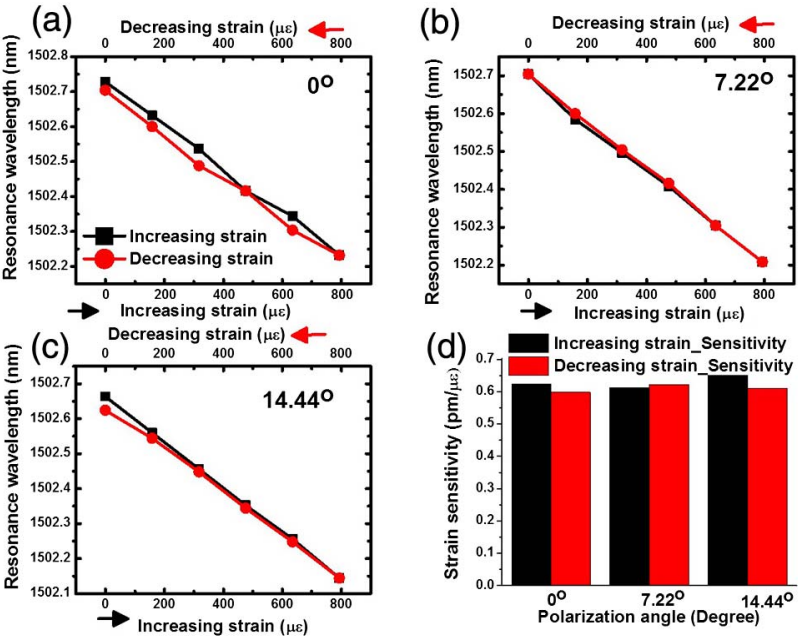

Fig. 8. Selected WGM resonances during the strain increase (black) and strain decrease (red) cycles with different input light polarization. Here, linearly polarized input light is rotated by angle (a) $0^{\circ}$, (b) $7.22^{\circ}$, and (c) $14.44^{\circ}$, respectively. (d) Sensitivity of the increase and decrease cycles of the axial tensile strain.

\section{CONCLUSION}

In conclusion, we investigated the effect of mechanical strain tuning of the WGM resonances excited in a POF cylindricalMR. The light was evanescently coupled into the micro-cylinder from a standard single mode silica optical fiber taper fabricated by the micro heater brushing technique. WGMs observed in the fiber taper's transmission spectrum had a high extinction ratio of up to $19 \mathrm{~dB}$ and a $Q$-factor of up to $2 \times 10^{4}$. We experimentally demonstrated that the spectral positions of the WGM resonances shift linearly during the increase and decrease of the axial tensile strain applied to the micro-cylinder in the range of strains from 0 to $1746 \mu \varepsilon$ $(0.17 \%)$. The WGMs move toward shorter wavelengths with the increase of the applied strain and return to their initial positions when the strain is decreased. Tuning of the WGMs is observed independently of the input light polarization. The WGM's resonance strain sensitivity reaches $0.66 \mathrm{pm} / \mu \varepsilon$. Strain sensitivity during increasing and decreasing cycles of axial tensile strain shows small differences, possibly due to nonuniform deformation experienced by the strained POF MR. The proposed POF-based strain tunable MR possesses several desirable features, such as ease of fabrication and modification of the resonator surface for potential applications in fiber optic sensing and tunable micro lasing.

Funding. Fiosraigh Dean of Graduate Students scholarship, Dublin Institute of Technology.

\section{REFERENCES}

1. K. J. Vahala, "Optical microcavities," Nature 424, 839-846 (2003).

2. M. R. Foreman, J. D. Swaim, and F. Vollmer, "Whispering gallery mode sensors," Adv. Opt. Photon. 7, 168-240 (2015).

3. M. L. Gorodetsky, A. A. Savchenkov, and V. S. Ilchenko, "Ultimate $Q$ of optical microsphere resonators," Opt. Lett. 21, 453-455 (1996).
4. V. B. Braginsky, M. L. Gorodetsky, and V. S. Ilchenko, "Quality factor and nonlinear properties of optical whispering gallery modes," Phys. Lett. A 137, 393-397 (1989).

5. Y. Louyer, D. Meschede, and A. Rauschenbeutel, "Tunable whispering-gallery-mode resonators for cavity quantum electrodynamics," Phys. Rev. A 72, 031801(R) (2005).

6. V. Sandoghdar, F. Treussart, J. Hare, V. Lefèvre-Seguin, J.-M. Raimond, and S. Haroche, "Very low threshold whispering-gallerymode microsphere laser," Phys. Rev. A 54, R1777-R1780 (1996).

7. T. Yoshie, L. Tang, and S. Y. Su, "Optical microcavity: sensing down to single molecules and atoms," Sensors 11, 1972-1991 (2011).

8. A. Ksendzov and Y. Lin, "Integrated optics ring-resonator sensors for protein detection," Opt. Lett. 30, 3344-3346 (2005).

9. S. Arnold, M. Khoshsima, I. Teraoka, S. Holler, and F. Vollmer, "Shift of whispering-gallery modes in microspheres by protein adsorption," Opt. Lett. 28, 272-274 (2003).

10. A. E. Fomin and M. L. Gorodetsky, "Spheroidal microresonators for the optoelectronics," Proc. SPIE 5948, 594818 (2005).

11. J. U. Fürst, D. V. Strekalov, D. Elser, A. Aiello, U. L. Andersen, C. Marquardt, and G. Leuchs, "Quantum light from a whisperinggallery-mode disk resonator," Phys. Rev. Lett. 106, 113901 (2011).

12. M. Sumetsky, "Mode localization and the $Q$-factor of a cylindrical microresonator," Opt. Lett. 35, 2385-2387 (2010).

13. A. Boleininger, T. Lake, S. Hami, and C. Vallance, "Whispering gallery modes in standard optical fibers for fiber profiling measurments and sensing of unlabelled chemical species," Sensors 10, 1765-1781 (2010).

14. G. Farca, S. I. Shopova, and A. T. Rosenberger, "Cavity-enhanced laser absorption spectroscopy using microresonator whispering gallery modes," Opt. Express 15, 17443-17448 (2007).

15. R. S. Moirangthem and A. Erbe, "Interfacial refractive index sensing using visible-excited intrinsic zinc oxide photoluminescence coupled to whispering gallery modes," Appl. Phys. Lett. 103, 051108 (2013).

16. V. S. Ilchenko, P. S. Volikov, V. L. Velichansky, F. Treussart, V. Lefevre-Seguin, J. M. Raimond, and S. Haroche, "Strain-tunable high-Q optical microsphere resonator," Opt. Commun. 145, 86-90 (1998).

17. R. Henze, T. Seifert, J. Ward, and O. Benson, "Tuning whispering gallery modes using internal aerostatic pressure," Opt. Lett. 36, 45364538 (2011).

18. T. Ioppolo, U. Ayaz, and M. V. Ötügen, "Tuning of whispering gallery modes of spherical resonators using an external electric field," Opt. Express 17, 16465-16479 (2009).

19. M. Agarwal and I. Teraoka, "Mode latching and self tuning of whispering gallery modes in a stand-alone silica microsphere," Appl. Phys. Lett. 101, 251105 (2012).

20. A. L. Huston and J. D. Eversole, "Strain-sensitive elastic scattering from cylinders," Opt. Lett. 18, 1104-1106 (1993).

21. T. Ioppolo, M. Kozhevnikov, V. Stepaniuk, M. V. Ötügen, and V. Sheverev, "Micro-optical force sensor concept based on whispering gallery mode resonators," Appl. Opt. 47, 3009-3014 (2008).

22. T. Ioppolo, U. K. Ayaz, and M. V. Ötügen, "High-resolution force sensors based on morphology dependent optical resonance of polymeric spheres," J. Appl. Phys. 105, 013535 (2009).

23. T. loppolo and M. V. Ötügen, "Pressure tuning of whispering gallery mode resonators," J. Opt. Soc. Am. B 24, 2721-2726 (2007).

24. U. K. Ayaz, T. Ioppolo, and M. V. Ötügen, "Wall shear stress sensor based on the optical resonances of dielectric microspheres," Meas. Sci. Technol. 22, 075203 (2011).

25. C. L. Linslal, M. Kailasnath, S. Mathew, T. K. Nideep, P. Radhakrishnan, V. P. N. Nampoori, and C. P. G. Vallabhan, "Tuning whispering gallery lasing modes from polymer fibers under tensile strain," Opt. Lett. 41, 551-554 (2016).

26. A. Mahmood, V. Kavungal, S. S. Ahmed, G. Farrell, and Y. Semenova, "Magnetic-field sensor based on whispering gallery modes in photonic crystal fiber infiltrated with magnetic fluid," Opt. Lett. 40, 4983-4986 (2015).

27. W. Lin, H. Zhang, B. Liu, B. Song, Y. Li, C. Yang, and Y. Liu, "Lasertuned whispering gallery modes in a solid-core microstructured optical fibre integrated with magnetic fluids," Sci. Rep. 5, 17791 (2015). 
28. J. C. Knight, G. Cheung, F. Jacques, and T. A. Birks, "Phase-matched excitation of whispering-gallery-mode resonance by a fiber taper," Opt. Lett. 22, 1129-1131 (1997).

29. G. Brambilla, V. Finazzi, and D. Richardson, "Ultra-low-loss optical fiber nanotapers," Opt. Express 12, 2258-2263 (2004).

30. W. von Klitzing, R. Long, V. S. Ilchenko, J. Hare, and V. LefèvreSeguin, "Frequency tuning of the whispering-gallery modes of silica microspheres for cavity quantum electrodynamics and spectroscopy," Opt. Lett. 26, 166-168 (2001).

31. R. Madugani, Y. Yang, J. M. Ward, J. D. Riordan, S. Coppola, V. Vespini, S. Grilli, A. Finizio, P. Ferraro, and S. N. Chormaic,
"Terahertz tuning of whispering gallery modes in a PDMS standalone, stretchable microsphere," Opt. Lett. 37, 4762-4764 (2012).

32. J. Huang, X. Lan, H. Wang, L. Yuan, T. Wei, Z. Gao, and H. Xiao, "Polymer optical fiber for large strain measurement based on multimode interference," Opt. Lett. 37, 4308-4310 (2012).

33. G. N. Greaves, A. L. Greer, R. S. Lakes, and T. Rouxel, "Poisson's ratio and modern materials," Nat. Mater. 10, 823-837 (2011).

34. R. Ulrich and A. Simon, "Polarization optics of twisted single-mode fibers," Appl. Opt. 18, 2241-2251 (1979).

35. D. C. Brown and H. J. Hoffman, "Thermal, stress, and thermo-optic effects in high average power double-clad silica fiber laser," IEEE J. Quantum Electron. 37, 207-217 (2001). 